\title{
Zur Perforation des Warzenfortsatzes
}

\author{
von \\ Dr. L. Jacoby, \\ prakt. Arzt zu Breslau.
}

Eiteransammlungen innerhalb des Warzentortsatzes und eitrige Schmelzungs-Processe desselben, der Trommelhöhlenwände oder anderer Theile des Felsenbeins sind durch die Näbe der Meningen, des Gehirns, der Acusticus-Ausbreitung; des Facialis, grosser venöser und arterieller Gefäss-Stämme, beziebentlich die Leichtigkeit der Uebertragung deletärer pathologischer Processe auf die genannten Nachbarorgane Zustände von der höchsten prognostischen Bedeutung. Acute eitrige Meningitis, Hirn-Abscess, Zerstörung des Labyrinths, Blutungen aus der Carotis oder der Vena jugularis, eitrige Thrombose mit consecutiver Pyämie sind als nicht allzuseltene Folgezustände in der Literatur*) verzeichnet.

Vermittelt wird die leichte Uebertragung der zu Grunde liegen. don Entzündungs-Vorgänge in der Richtung nach den Meningen und dem Gehirn durch den Reichthum der betreffenden Knochen an Gängen und Spalten, die mit Bindegewobe ausgekleidet sind, die bindegewebigen Hüllen vieler Gefässe und Nerven; die Zerstörungen des Labyrinths, die Anätzung der grossen Gefässstämme durch die caustisehe Beschaffunbeit des Eiters und die namentlich im kindlichen Alter nieht selten papierdünnen oder defecten Scheidewände; endlich die eiterige Thrombose durch die erfahrungsgemäss mit Leichtigkeit

*) Vide dieselbe insbesondere bei Tröltsch, in Handbucbe der allgem. und speciellen Chirurgie, herausgegeben ron Pitha und Billroth. 1866. III. Bd. 1. Abth. 2. Lief, S. 45 u. 49, sowie bei Moos, Klinik der Olirenkrankh, 1866. S. 232-81. 
zu Stande kommende Thrombenbildung in den Knockenvenen der spongiösen Substanz. - Gegen die crstgenannter Zustände (Eiteransammlung und (aries) bat sich die An- beziehentlich Durchbohrung des Warzenfortsatzes als oin ebenso rationelles wie bewährtes Heilmittel orwiesen. Von den bisher in der Literatur verzeichneten 15 Operations-Fallen (einschliesslich des meinigen) sind nur vier lethal verlaufen durch Umstände, die dem operativen Eingriff selbst unzugäuglich waren. - Nach James Hinton (Medical Times and Gazette, 26. Sept. 1868) haben ron 14, von verschiedenen Autoren berichte. ten, Fallen 12 einen glicklichen Ausgang gehabt, und ron den zwei ungünstig verlaufenen war in dem einen zar Zeit der Ausführung der Operation schon Pyämie vorhanden.

Das therapeutische Verhältniss der fraglichen Operation an den mehrfach genannten Krankheits-Processen beruht zunïthss auf dem günstigen Einfluss, den anerkanntermassen Abscess-Eröffnungen im Allgemeinen auf den normalen Ablauf der primären Entzindungs-Vorgänge und die Erhaltung, resp. Wiederherstellung der Integrität der betheiligten Gewebe und Organe ausüben; bei vorhandener Caries auf der Möglichkeit ausgiebiger Bespülungen und der Anbringung zweckmässiger Medicamente. Die Nothwondigkeit der Abscess-Oeff nung aber tritt selbstredend unwiderstehlich ein da, wo die Stagnation des Eiters so unterstützt wird wie im Processus mastoideus (Reichthum an ron einander geschiedenen Höhlen und relativ bohe Lage der Communications- resp. Abflussöffnung nach der Trommelhöhle hin); in der Paukenhöhle bei nicht oder nur in geringem Umfange stattgehabter Perforation des Trommelfells (relativ hohe Lage der natürlichen Abluss-Oeffung, des Ostium tympanicum tubse zu dem tiefgelegenen Boden der Pauke und vielfache Buchten, besonders an der Labyrinth-Wand); in der spongiösen Substanz der Pyramide oberhalb der Trommelhöhle, dem gewöhnlichen Sizze der selbstständig und isolirt entwickelten Caries des Felsenbeins. -

Auf alle die soeben erörterten anatomischen Verhältnisse und die daraus hergeleitete therapeutische Beziehung der Operation zu den fraglichen Krankheitszuständen zuerst aufmerksam gomacht zu haben, ist das besondere Verdienst von v. Tröltsch (vd. Virchow's Archiv Bd. XXI p. 295, 1861), wo zugleich ein von ihm mit glucklichem Erfolge operirter Krankheitsfall referirt wird. Wer sich für die Geschichte der Operation vor dem Jahre. 1858, in welchem $v$. Tr. seine exste Beobachtung machte, interessirt, findet die bekreffenden Data in der soeben citirten Arbeit. Nach ihm wurde cine Reihe von mehr weniger instructiven Operations-Fullen veröfentlicht, die sich in chro- 
nologischer Ordnung in seinem Lehrbuch der Ohrenkrankheiten 1868 p. 384 verzeichnet finden. Unter ihnen sind von besonderem Interesse die Beobachtungen von Pagenstecher (Archiv für clinische Chirurgie Bd. IV p. 523, 1863) wegen ihrer wichtigen Notizen über die directe Fortleitung entrïndlicher und Eiterungs-Vorgänge von der Cutis des 3ussern Gehörgangs auf das Periost, resp. das' des Processus mast. und die Knochensubstanz selbst oder vom erstgenannten Orte ruf demselben. Wege zur spongiösen Substanz des Felsenbeins oberhalb der Trommelhöhle, wie wegen des Nachweises des Zusammenhanges zwigchen einer Anfangs nur die Cutis afficirenden Otitis externa und den bisweilen mit uberrasehender Sehnelligkeit, in Folge der durch anatomische Continuität vermittelten Theilnahme des Endocranium sich entwickelnden Carebral+Symptomen. Zu den 1. c. angeführten Fällen kommt zunächst noch die ron James Hinton (medical Times and Gazette 1. c.) veröffentlichte Beobachtung. -

Die bekannt gewordenen Fälle stellen bezüglich der Zeit, welche rom Beginn des Krankheits Processes bis zum Operations-Termine verfloss, der Localisation jenes sowie des Ortes und der Art der Ausführung der Operation noch kein besonders reichbaltiges Material dar. Deswegen und insbesondere weil einzelne wichtige Punkte, namentlich der für die Operation geeignetste Termin, der relative Werth Jor nicht operativen Beliandlung, die zur Ausführung der Operation beniitzbaren Instrumente einer Erörterung und genauern Feststellung mir nicht unwerth erschienen, habe ich mir erlaubt, die nachfolgende Beobachtung in extenso und die angeschlossenen Bemerkungen, einschliesslich der aus den bisherigen Fällen, soweit sie mir in der Li. teratur zugänglich waren, sich ergebenden Resultate zu veröffentlichen. -

Helene Berkowiz, 48 J, Sohuhmachers-Frau ron hier, stellt sich zum ersten Maìe vor 11./6. 1868 und erzählt, dass sie, seit eỉner Reihe ron Jahren bis zu ihrem Erkranken kräftig und gesund, in Folge einer manifesten Erkältung durch Zuglaft bei anhaltendem Aufenthalt an einem in der Nähe des Koehofens belegenen Fenster mit erhitztem Körper vor eirca noht Wooken nater heftigen linksseitigen Ohrenschmerzen erkrankt sei, denen sich alsbald solche in der Schläfen-, Hinlexhanpts- nd Hinterolrgegend, sowie subjective Geränsche (Ruusehen) beigesellten. Die Schmerzen! seien in wenigen Tagen un einer fast unerträglichen Höhe gektiegen, hattien sich, nachdem es zum Erguss einer eitrigen Flïssigkeit aus dem Gehörgange gekormmen war, awar oinigermassen ermässigt, wïren aber, einschliesslich der irradiiten wad insbesondere der Hinterohrschmerzen wie anch der sabjectiten Gexäusohe von $d a$ ab constant and ziemlid gleiehmässig bis zu ibrer Meldung

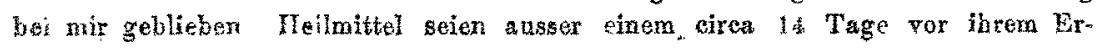


zeheinen bel mir auf den Processus mastoideus gelegten Vesicator und einem allgemoinen lanen Bade keine angewexdet worden.

Die objective Untersuchung ergab: Linkerseits Uhr nur beim Anlegen an die Ohrmuschel; nur ganz laut gesprochene Zahlen abgewandt auf etwa 4 . Entfirnung: Stimmgabel rom Sohádel ans nur links. Die Cutis des Processus mast. war mässig geröthet, in geringem Grade geschwollen und der Knochen selbst auf Druck in die Tiefe sehr empfindlich. Die schon erwähnte Vesicator-Wunde war seit einer Reihe von Tagen geheit und dis Epidermis unversehrt. Der Meatus auditorius war durch eine von der hintern Wand ausgehende schmexkhafte Schwellung so verengt, dass ein Trichter nicht eingebracht werden konnte. Nichtsdestoweniger erkannte man nach Anwendung ron Politaer's Verfahren (wobei deutjiches Perforations-Geriusch wahrnehmbar war) und reinigenden Wasser-Einspritzungen eine kileine Perforation in dem rordern mittieren Absobnits des Trommelfells. Eiter warde in sehe mässiger Quantität durch das angedeutete Verfahren herausbefördert und fon der Pathentin wie ihrem Manne mitgetheilt, dass die Eiterung stets nar gering gewesen sei. -

Auf Grund der iberwiegenden Betheiligung des Processus mast. an dem vorliegenden Entzindungs. Processe des Mittelohres warde sofort cin ungefäht 3/4" langer Einschnitt auf denselben bis auf den Knochen nach Wilde'scher Vorschrift gemacht, đurch den man die Knochendecke selbst noch vollkommen hart und glatt fühlte. Die beiläufig solchergestalt veranlasste ziemlich ergiebige Bintang wurde eine Zeit lang durch warmes Wasser unterhalten, ein mässig antiphlogistisches Regime, Fortgebrauch des habituell benutzten kalten Sennesblätter-Aufgusses und zum eventallen Gebranch Morphium für den Abend verordinet.

Bei meinem Besuche am 12./6. referirte die Kr., dass sie in der Nacht zwar besser geschlafen, die Intensität der Sehmerzen sich anch ermässigt habe, dase dieselben aber immer noch in erklecklichem Grade fortbesteben. Im Uebrigen war eine Reaction auf das Verfahren nicht eingetroten, der Puls zähite einige nnd 70 Schläge; das soustige Untereuchungs-Resultat glich durchaus dem gestrigen. Ord.: Ruhe, mässig nährende, nicht congestionirende Diät, Fö:derung der Evacnatio alvi, Ohrbäder, Kataplasmen, reinigende Injectionen, eventuell Morphium.

13./6. Symptomatiscin: status idea. Ord, : Fortsetzung des gestrigen Verfahrens und Tinct. Jodi in der Nahe des Proc, mast. täglich oder seltener in bo schränktem Umiange einzupinseln; versuchsweise Solutio argenti nitrici c. 0,6-90,0 mit nachträglicher Neutralisirung und vorangängigem Politzer'schen Verfahren, einmal täglich 15 Tropfen in den M. a. zu instilliren.

14./6. Die Schmerzen hatten, ob ix Folge der adstringirenden Solution oder aus einem andem Grunde, mag dahingestell bleiben, etwas zugenommen. Dex Schlaf war mehr gestört; desswegen wurde die Sol, argenti nitr. fortgelassen, ausser den obigen Verordnungen noch 3 firudine hinter das $\mathrm{Ohr}$ nd Electuarium leitivum bis zu rejchlicher Wirkung empfohlen.

15./6. Status idem. Ordinai. eadem mit Ausnahme der Hirudines und der Purgans.

16./6. Status idem, aber Abnahme dor irracititen Schmerzen. Ordination uzverändert.

17.6. Znnahme der versehiedenen Schmerwen. Ordination: Hirudinec tres binter das Oh: und 2 stibdNch 1 Gran Calomel,

18./6. Ermässignmg der Schmenzen. Calomel continuctur. 
19/6. Furunkel der vordern Partie der hintem Gehörgangswand. Oräination: flelssige Benützung der Ohrbüter und Calomel anest tzen.

20/6. Status idem. Ordination: 2 Hirudines hinder jas ohr.

Am 23./6. wurden zam ersten Male menrere kleine gesehwellte Lymphdrïzer in der Richtung des Sternoeleidomastoidens wahrgenommen.

26.16. wieder 2 Blutegel hinter das Ohr.

Vóm 29./6, wurdea, wegen Zunahme der Schmerzen in der Tiefe des Ohres, der irradirten und der Hinterohrschmerzen regelmässig einen Tag zweì Bíutegel, den näehsten Tinet. Jodi applicirt und mit den sonstigen purifieirenden und calmirenden Verordnungen fortgefahren; am 12./7. wurde in der Temporal-Gegend eine aubcutane Morphium-Injection (1/s Gran) gemacht, Darauf sehwanden die Temporal. Schmerzen, die übrigen blieben unFerändert, Schlaf trat nicht ein. In den nächsten Tagen wurde die Dosis des subcutan injicirten Morphiums etwas erhöht mit etwas mehr Linderung der subjectiven Beschwerden,

19./7. Patientin hat bcim Autstehen oinaz epleptitschen Anfall gehabs; es bleibe aber zweifehaft, ob derselbe mit dom gegenwärtigen Krankheis-Processe in Verbindung za bringen ist, weil in langen $Z$ wischerräbmen auch sonst vereinzelte Anfälle da waren. Infolge dessen wurden methodiseh nach Art einer Inunctions-Cur joủer 2waiten Tag 2,0. Unguentum hydrargyri ein. eingerieben und am freien Tage 2 Blutegel applicirt.

Am 24./\% hatte die Eiterabsonderung ats dem Gehörgang so erheblich abgenommen, dass ich mich veranlasst sah, an diesem und dem folgenden Tage anf einige Zeit Pressschwamm in denselben einzulegen, die Fiterentleerung aus der Tiefe wurde dadurch zwar etwas reichlicher, die Schmerzen aber ermüssigten sich nicht wesentich. Die Empfindlichkeit des Processus mast. hatte unter dem methodischer Gebrauche des Ungt. cinereum und der Jod-Tinctar allmälig erheblich sich vermindert. 'Nur die hintere untere Partie war noch mässig emptindlich geblieben. Ein Druck auf dieselbe steigerte von jetat ab regelmässig, so oft das Experiment auch wiederholt werden mochte, die subjectiven Gerdusche; es entstand sofort neben dem gewöhnliehen Rauschen noch ein pfeifendes Gerăgsol. - Auch anf der rechten Seite schwellen die Lymphdrisen lings der obern Halfte des Sternocleidomastoidens allmälig an, so, dass theils desswegen theils wegen beginnender Stomatitis mercurialis "die Inunctionen vorübergehend ausgesetzt warden und Kali chloricum in bekannter Weise ordinirt wurde.

Nachdem das oben dètallirte Verfahren, Kirudines, Unguentum cinereum. Tinct. Jodi mit periodischen Unterbrechungen bis zum 10./8. fortgeführt war, war die $\mathbf{K r}$. unter gleichzeitigem Eintreten der Menses subjectiv schmerzfrei, das Verhaiten des Processus mast. für die objective Untersuchung aber gleich dem zuletat besciniebenen und die Eiterabsonderung ans der Tiefe des Obres auf ein Minimum reducirt. Dem entsprechend wurden die obengenaninten Medicamente seltner und in geringerer Dosis angewandt. Die soeben bezeichnete Besserung hielt mit unwesentlichen periodischen Verschlimmerungen an bis zum 2./9., wo ich die Kranke in Folge einer Reise auf mehrere Wochen verliess. -

Bald nach meiner Abreise änderte sich laut Mitheilung des mich vertretenden Collegen und der. Kranken die Scene. Trotz der in angemessenem Unfange fortgesetzten Antiphlogose nahmen die örtlichen und irradiirien Schmerzen, die Störungen des Seblafes und subjectiven Geräusche zu; der. Warzenfortsatz gewann an Umfang, die Weichtheile oberhalb desselben wurden nach und nach infiltrirt and es fand allmälig die Bildang eines Lleinen Abscesses in denselben statt, - Leider 
weigerte sich die $\mathrm{Kr}$, damais, auf die ihr Seitens des mïch rertretenden Collegèn rorgeschlagene Operation einzugehon.

Trotz der an 24./9. durch mich vorgenommenen Erweiterung der siebartigen Perforation der Weichthelle und obgleich jeh ungefähr $3 / 4$ " in der Richtung nach hinten und untea die Sonde vorsehieben konnte, war ich ausser Stande, eine Rauhigkeit der Knochenoberfäche beziehentlieh einen Durchbruch des Knochens zu entw decken. Nachdem durch reichhehe Eiterentleerang, subcntane Morphium-Injection und die bereits frither erwähnten Palliativ-Mittel der Zustand in solcher Weise noch bis zum 2./10. fortgedauert hatte (za. welcher Verzögerung änssere Umstände beitrngen, entschloss ich mich in Hinblicke auf die durch das bisherige Verbalten des Krankheits-Processes gegen die mit grösster Consequenz fortgesetzte Antiphlogose und die zur Genüge erwiesene Unmöglichkeit, die rorhandene Eiter-Retention und cariöse Schmelzung des Warzenfortsatzes anderweitig zu beseitigen, zur Perfomation desselben. (Der grösseren Genanigkeit wegen bemerko ich, dass bis zum 3.10 im Ganzen 83 Blutegel and 36 Grm. Ung. oin. verbraucht worden sima.) -

Die Operation selbst fand unter Assistenz meines Freundes Dr. Steuer in der Chloroform-Nareose Statt. Nachdem duroh die vielleicht $8 / 4-1^{\prime \prime}$ tief infiltrirten Weichtheile ein cirea $11 / g^{\prime \prime}$ langer Einschnitt bis auf den Knochen gemacht worden war, warde mittelst Tastens deutlich in der Richtung nach vorn und unten die iussere Fläche des Processus mastoideus ron Periost entblösst nnd rauh gefilihit. In der Näbe und etwas oberhalb dieser Stelle wurde der akidopeirastische Bohrer (Middeldorpf's) anfgesetzt nnd so lange in einer der aussern Oeffinung des Meatus suditorius parallelen Riohtung die ungefuhr auf $3^{\text {"I }}$ rorgeschraubte Bohrspitze in Bewegung gesetzt, bis das Gefihl des aufgehobenen Widerstandes das Eingedrungensein in die Knochenhöhie deatlinh manifestirte. Wie viel Eiter unmittelbar nach diesem Acte aus der etwa ein Paar Linien breiten Oeffnung der Wussern Knochendecke entleert wurde, war schwer zu bestimmen, da aus den stark infiltrirten Weichtheilen Eiter mit Biut gemischt während der ganzen Operationsdauer in reichlicher Menge herrordrang. - Nach Beendigung der Operation einfacher CharpieVerband, kalte Compressen, Bonillon.

Beim Abendbesuch war der Puls ruhig, keine erhöhte Hant-Temperatur zugegen, die Schmerzen hinter dem Ohre rerschwunden, aber die anf dem Scheitel noch vorbanden. Ordinat.: Fortsetzung der Epithemata frigida, solange sie der Kranken angenehm sind, Charpie-Wechsel, eventuell. Morphium.

4./10. Beim Abendbesuch ist der Puls bis 92 accelerirt; erhöhte Temperatur nicht wabrnehmbar. Die Schmerzen in der Scheitel- und Occipital-Gegend bestehen noch fort. Ordinat. wie gestern.

5./10. Bei dem heutigen Frithbesuche hat sie einen Puls von 90. Gestern Abend hat die $\mathrm{Kr}$. beim Heraussteigen aus dem Bette zehn Minuten etwa stark gefroren, nater lebhaften Träumen sehr unruhig geschlafen und klagt zur Zeit tiber allgemeine Schwere des Kopfes, Schmerzen in der Scheitel- und Occipitalgegend. Die Wundränder und der Grund der Wunde waren leicht eitrig beschlagen; von normalen Farbenverhältnissen, die Umgegend war unzweideutig abgeschwollen und auf Druck viel weniger empfindlich als fruher. Es verbleibt zaher bei mild nährender Kost und einfachem Charpie-Perbande. Beim Abendbesuch ist-dex Puls wieder auf 80 zurückgegangen.

6./10. In der Whe der früheren spontanen Darohbruchstelle hat sich ein kleiner Abscess gebildet, der mit Frleichterung eröffnet wird. Im Uebrigen symptomatiseh und beztiglich der Ordination keine Aenderung: Abends kein Fieber. 
Von 10./10. ab werden Charpiewioken bis in die Knochenwunde gingemihr. So geht es fort bis zum 12./10. Wax auch die Kranke arach der Operation bis jetzt nicht frei von Schmerzen; so war doeb der Zustand des Kopfes naoh ihrer mehrfachen Versicherang nm vieles erträglicher unả angenełmex gewesen alą vorber. $D_{a}$ tritt Wuad-Erysipel mit starker Schwelung der Wanduänder, Frostanfall, Hitze, Benommenheit des Kopies und Uebelkeiten efn. Puls 100. Ordination: 2stürdlich 2 gr. Calomel.

13/10; Anf wiederbolte Calomel-8tühle ist Puls Abends wesentheh rahiger, Temperatur günetiger, Kopf 'freier, abei noch Debelkeiten vorhanden.

14/10. Der Schlaf der letzten Nacht war ein sehr urruhiger, Puls 120, wenig erböhte Haut-'Temperatur, das Erysipel nimmt die ganze linke Gesichtshilite und einen grossen Theil der linksseitigyen Kopfhaut ein; 2 malige Defacation auf ein lñfasum Sennae dagewesen.

15./10. Den ganzen Tag viel Delirien. Puls Morgens 110; mässige Temperatur-Erhbung. Abends: Puls 120. Das Erysipel ist bis auf die andere Gesichts. bälfte gewandert. Morgens und Nachmittags sind viel dänne Stihle dageweser, viel kaltes Wasser wird verabreicht.

16:10. Die letate Nacht war dureh blande Dolicien und Stöhnen sebr unruhig; Morgens war ein hochgradiger Collapsus eingetreten, so dass alle sehnell zu beschaffenden Reizmittel, Kaffee, Aether, Bouillon angewandt werden mussten: Der Tag wurde zum grössten Theil durch Delirien ausgefüllt. Urin geht wnwillkürlich ab. Ord.: Hänfge Verubreichung kleiner Portionen Bouillon und Acidi miriat. 1,25 solut. in aquae deet 150,0 , 2stündich 1 Esslöffel. Abends oventuell Campher.

17./10. Puls Abends circa 115; sich selbst tuberlassen delirirt die Kr, mehr weniger lebhaft. Ordination: fir die Nacht zwei Campher-Pulyer $a, 06$.

18./10. Die Delirien bestehen noch fort. Nachmittags ist die Kr. ruhiger. Pulsus filiformis. Mit Rutoksicht auf die melurnächtige Schlafosigkeit etc, Ordination: 40 Tropfen Tinct. opii crocata mit 20 Tropfen Aether gemischt, Den 4 ten Theil als Einxelgabe zstünaliah verabreicht.

19./10. 2 Dosen der Opium Tinctur sind ohne alle Wirkung geblieben und mehr irthitmlielew. Weise nicht verabreicht; die Kranke bat fort und fort delirirt; Nachts angeblich $3 / 4$ Stunden gefroren und dann mässige Hitze bekommen. In der linken Axillar-Gegend fanden sich mehrere acut infiltrirte Lymphdrísen; das Erysipel erstreckt sich bis auf die Mitte des Ritckens, namentlich stark eatwickelt in dem Interscapular-Raume, - Bein Abendbesuch ist der Zustand bezüglich der Hirnfunction and dos Tiebers derselbe.

20./10. Ordination: 20 Tropfen Tinct. opii crosat., eventuell nach einer balben Stunde noeh 10. Tropten. - Auf diese beiden Dosen trat Ruhe und achtzelnstiundiger fast ununterbrochener Sehlaf ein; beim Abendbesweh ist die Kr. bei Besinnung und delirirt nicht mehr. Ordination: Nur im Falle wiederkehrendex Delirien noch 10 Tropfen Tinct, opii.

21./10. Delirien haben aufgehort. Nachts hat sie mit Auswahma von $21 / 2$ Stunden geschlafen, Puls 100, reichlicher Schwoiss. An der rechten Seite des Halses und Nackens Erythem und periodischeZuckungen der rechtseitigen Ober-Extremität. bisweilen anch der untern. Ordination: Ungt. cinerenm fitr die rechte seite des Halses.

22./10. Nachts: Seblaf. Erythem und rejhtsseilige acute Driksen - Inflitate bestehen boch fort. Pnis Abends: 100. Sensorinon vollizommen frei, roehrere dünrAússio Defăcationen per diem. 
Vom 23./10. bis 7./11, hahen sith die Erytheme allmälig verloren, die Infltrate in der Submaxillar und den Axllar-Drüsen bestehen noch fort; die Operations-Wunde, beziehentheh die Stelle des spontanen Durchbruehs eitern reichlieh; die Kopf-Symptome nenmen allahlig $n$; ; cs hat sich ein atwa achtgroschenstückgrosser Decubitus am Os sacrum eingestells. Der Puls ist am 7./11. bis anf 80 heruntergegangen. Ordination: Ferrum cartonieum saecharat. Speckeinreibungen; für die Drüsen-Inflitrate: Inunetion kleiner Dosen ron Ungt. Kalii jodati, reiehliche nahrhafte leichtrerdaulicke Kost. -

20./1. ist der Kops ganz frei, die Kräfte nelumen zu, die Operations-Wunde eitert mässig; die subjectiven Gerüuscho verliercn allmallig ihre Intensität; der Decubitus heilt; die Submaxillar- und Axillarirüsen abscediren allnälig ond werden arn 27./11. unä $90 . / 11$. geöfínet.

Am a./12. wurden als Zeichen unvolkommener linksseitiger Facialis-Lähmung bemerkt: Unmöglichkeit, die linke Seite der Stirn zu runzeln und Nichtbewegen der linken Hälite des Mundes beim Sprechen. Schon am 3./12. verliert sith die Parese des linken Frontalis; die des linken Mundwinkels besteht nock fort; die Operations-Wunde eitert sehr mässig; aber man erreicht mit der Sonde den kranken Knochen nicht mehr; die rerschiodenen Abseesse eitern mässig; der Decubitus ist zugeheilt; die Kräfte kehren langsan zurüe ; Digestion, schlaf, snbjecti\% es Verhalten des Kopfes durchans ginstig.

7./12. Die Parese des linken Mundwinirols besteht noch fort, dis subjectrven Geräusehe halen tageweise aufgehört, kehren aber bisweilen zurick.

9.12. Bei Untersuchung mittelst Spiegel und Trichter sieht, man deutlich eine kleine Perforation im vordern mittlern Trommelfell-Abschnitte; bei Politzer's Vorfahren dringt die Luft subjectiv fühlbar hindureh.

22./12. Die Wunden auf dem Processus mast. sind vollständig vernarbt; links besteht noch ein kleines fistulöses Axillardrüsengeschwür und rechts ein solches der Submaxillardrüse. Die Reconvalescentin hört, die Ubr links ungefuhr 11/2" (rechts etwa $4^{M}$ ) und sebr leise gesproohene Zahlen abgewandt nugetshr anf $10^{f}$ Entfernung; subjective Geräusche (Pfeifsn) nur noch periodisch und selten; die wichtigsten Functionen normal, nur noch Schwähhe der Unter-Extremitäten; die irthere Kranke ist in ihrer Wirthschaft wieder thätig. Ordinat. : Roborirende Diut und Fortgebrauch des Eisens, -

27./12. Die Reconvalescentin klagt heute wieder über etwas Hinterkopfschmerz, der schon seit längerer Zeit verschwunden war, unruhigern Schlaf und vermehrte subjective Ceräusehe, muthmasslich Folgen zu angesirengter erbitzender Arbeit (am Kochofen) und der bevorstehenden Menstruation. Die Parese des Mundwinkels besteht. Beztiglich der Energie des Gehörs ist das Verhalten wie 22.12. Die Knochenleitung für die Uhr ist $R$. wie $L$. sehr prompty normal, nur ₹orn linken Processus mast. etwas schwächer. (Bei dar Aufnahme der Kranken ist in der Eilo das Verhalten der Knoobenleitung nicht notirt worden.)

29.12. Die smbjectiven Geräusche sind wieder verschwonden, der Schlaf war ruhig. Die Hinterkopfsebmerzen rechterseits haben sich ermässigt; die Parese des linken Mundwinkels iat unverändert. 


\section{Epikrise mond Aesultate aus den bisherigen Beobactungen.}

Der der Kranken am 1. Consultations-Tage safart vorschriftsmässig gemachte Wilde'sche Einschnitt brachte trotz roichlicher Blntu ung nur unerbebliche Erleichterung. Da aber die oberfachliche Knochensebicht noch sehr fest sich anfühlte und dringende Symptome nicht vorhanden waren, so erschion mir zunä̉chst für die weitere $\mathrm{Bo}$ handlung örtliche Antphplogose allein indicirt. Wenn ich dieselbe bis Anfangs September und in grösserom Umfange anwandte, als dics wohl sonst zu geschehen pflegt, so bestimmten mich hiezu folgende Umstände: Antiphlogistische Mittel waren bis zum Beginne meinex Behandlung noch nicht in Gebrauch gezogen, die Toleranz der Kranken war der Anwendung derselben günstig; es wurden rorübergebend wenigstens subjectir gute Erfolge erzielt; es waren endlich Erscheinungen, die einen eitrigen Schmelzungsprocess des Knochens: manifesm tirt hätten, nicht zugegen (negratives Resultat der mikroskopischen Untersuchung und negatives Verhalten der Nachbarschaft mit Ausnahme einiger Lymphdrüsenanschwellungen). (Hiezu kommt, dass nach einex mündlichen Mittheilung meines verehrten Collegen Schwartze in eincelnen Fullen beginnende derartige Processe im Knochengewebe des Warzenfortsatzes durch consequente Antiphlogose geheilt werden, wobei allerdings dahin gestellt bleibt, ob dieser an sich gewiss richtige Sałz noch auf einen bereits seit acht Wooben bestehenden Vorgang Anwendung finden darf.) Endlich sprach auch eine Umschau in der mir zu Gebote stehenden Literatur mittelbar für die einstweiligo Unterlassung der Operation.

Die bisher veröffentlichten Fälle zerfallen bezüglich des Operations-Termins und der begleitenden resp. bestimmenden Umstände in zwei Reihen:

A) solche, in denen operirt wurde wegen hochgradiger entzündlicher Vorgänge in der obcrflächlichen Schichte des Processus mast, dic den primitren acuten eitrigon Trommelböblen-Katarrh begleiteten oder sich ihm anschlossen;

B) solche, wo entzindliche Erscheinungen, beziehentlich Zeiched eitriger Schmelzung in der obertächlichen Knochenschichte mebr oder weniger fehlten.

Zux ersten Reihe gebören der Fall von $v$. Trötsch (Virchow's Archir 1. c.); Tumbull's 3 Falle (The med, and surg. Reportex. Philadelphia Febr. 15. 22. 1862); in Fall von Schoartze (prakt. Beiträge zur Ohrenheilkunde, Würzburg 1864 p. 37); zmei Beobacht- 
ungen ron Pagenstecher (Archiv für klin. Chirurgiel. c.); der Maier'sche Fall (dieses Archiv I. 226); die drei Kessel'schen Fälle (Inauguralaissert. Giessen 1866. Fäle von Otitis int. mit Vereiterung der Zellen des Warzenfortsatzes, Sinus-Thrombose. Perfor. des Warzenf.); $J$. Binton's Fall (l. c.); endlich meine Beobachtung.

Zur zweiten Reihe gehören die Falle von Flaiz (dieses Archiv 1I. 228) und die erste Beobachtung ron Pagenstecher (Archiv für klinische Chirurgie 1, e.).

Ad A. 1) v. Tröltsch operirte ungefăhr 4 Wochen nach dem Beginn des acuten eitrigen Trommelhöhlenkatarrh's wegen schneller Steigerung der entzündlichen Vorgänge im Warzenfortsatze bei gleichzeitigern Fieber, immer quälender werdenden Schmerzen und bedrohlichen Erscheinungen, welche eine Weiterleitung des Processes rom Ohr auf die Schädelböhle fürchten liesson.

2) Turnbull (1. c). In ersten Fall entstand, ohne dass gleichzeitig Eiterausfluss aus dem Meatus auditorius bestand, ungcfabr 4 Wochen nach dem Beginn des Scharlachfiebers eine acute, sehr schmerzhafte, hochgradige Schwellung des Proc. mast. mit imminenten CerebralSymptonen und Fieber, nachdem zur Zeit der Abnahme des Eruptionsfebers schon eine Otitis externa rorangegangen war. Wilde'sche Incision resp. Durchschneidung des Periost's, reichlicher Eitcrerguss, Schluss der Wunde nach drei Wochen; Vesicans; noch 4 Wochen lang: unterbaltene Eiterung; Heilung mit Depression des Proc. mast. -

2ter Fall: eine 3jjäbrige Fran ist, nachdem sie 3 Wochen Sym. ptome einer durch Erkältung entstandenen acuten Otitis media gehabt, bei ihrer Aufnahme in das Hospital rechterseits schwerhörig. Obrmuschel, Meatus auditorius, Hinterohrgegend sind intensiv entzündet, T.-F.Perforation fehli; Fieber. - Antiphlogose mit Erleichterung nach wenigen Tagen; die Schwellung in der Gegrend des Proc. mast. besteht noch fort; hiezu hat sich ein geringer Ausfuss aus dem Gehörgang hinzugesellt. Dabei bestehen sehr heftige periodische Scbmorzen fort. - Incision bis auf den Knochen mit Biterentleerung am nächstén Tage und Schwinden der entzündlichen Local-Symptome. Einige Monate später ein Recidiv des örtlichen Vorganges am Proc. mast., Erwciterung der noch nicht verheilten Knochenwunde, Unterhaltung der Kiterung, Losstossung der erweichten oberfläeblicken Knochenschiaht und Heilung nach mehreren Wochen mit Depression dus Knochens.

3ter Fall: Mädchen von 8 Jahren, im September 1861 in das Hospital aufgenommen. Nach bösartigem Scharlach Schwerhörigkeit, jauchiger Ausfluss aus dem rechten Ohre; in der Nähe des Trommer 
felles Granulationen; das Trommelfell selbst $3 / 4$ zerstört; verlässt gebessert das Hospital. Am 21. September wird Turnbull eiligst gerufen. Nach einer Erkaltung durch Zugluft haben sich Nachts beftige Schmerzen hinter dem Ohre, Kopfschmerzen, Schlaflosigkeit, Frost mit Hitze wechselnd und starke Schwellung des Proc. mast. eingestellt, die sich bis in das Gesicht und zu den Augen ausdehnt. - Tiefe Incision, reichlicho Blutung, Kataplasmen, Antiphlogose. Im November: Durchbohrung der oberfizchlichen Knochenschichte mit einer scharfen Houlsonde und Application ron Argentum nitricum, um die Eiterung reichlicher zu machen. Im December: Brweiterung der Knochenwunde und Entfernung eines schmalen Knochenstizcks. Injection schwacher Zinklösung. Am 19. December: Entfernung eines breiten Sequester's dessen Beweglichkeit vorher dureh dic Sonde festgestellt war, nach Erweiterung der Wunde. Schon 2 Tage nachher beilt die Wunde mit Depression des Knochens.

3) Schwartze: Circa 8 Tage nach Beginn des Eiterausflusses aus dem linken Ohre wird mittelst Sonde leicht die rauhe, oberflächliche bloshegende Knochendecko durchbrochen, sofort reichlich Eiter entleert, nachdem mehrere Tage vorhor durch Wildesche Incision ein Abscess der Weichthelle mit reichlicher Eiterentleerung eróffnet war.

4) Pagenstecher's erster Fall (im Texte) (60jähriger Buchbinder) 1. c. p. 525. Eitriger Ohrenfluss seit 8 oder 10 Tagen; seit einigen Tagen Betäubung und intensive Entzïndung des Proc. mast. Incision und Durchdringung der Knoehendecke mittelst Kuorpelmessers. Eiterentleerung und schnelle Heilung bei unversehrtem Trommelfeli.

Pagensteches's 2. Beobachtung 1. c. pag. 533. Ungefähr 5/2 Monat nach dem Beginn eines acuten eitrigen Trommelhohlen-Katarrhs mit Perforation des Trommelfells und Facialis-Lähmung entwickeln sich die Erscheinungen intensiver Entzüudung. des Proc. mast, mit cerebralen Symptomen, 2 Tage nachber (im Mai) Anbohrung desselben dicht aber der Begrenzungslinie des Gehörgangs und in der Richtung der Pyramide. Heilung bis zum September mit Wiederberstellung leidlichen Gehörs und zurückbleibender Facialis-Lähmung.

5) Der Fall von James Hinton. Eröffnung des Pros. mast. mittelst Troiscarts nach vorangängiger Incision wegen bochgradiger schmerzhafter Schwellung desselben und bereits stattindender Erweichung der oberflächlichen Knochenschicht. Reichliche Eiterentleerung mit grosser Erleichterung. Heilung der Wunde nach 5 Wochen. Recidiv-Eröffnung eines grossen Abscesses unter dem Sternocleidomastoideus, grosse Erleichterung der Kopfbesehwerden mit jedesmaliger Rückkehr derselben, sobald der Eitorabfluss stockt. Vollstïndige Frei- 
logung des Abscesses nach 3-4 Wochen und definitive Heilung nit Wiederherstellung des Gehörs.

6) Der Maier'sehe Fall. Nach 15monatlichem schmerzlnsen Bestehen ciner fôtiden Otorrhoe acute hochgradige entzündliche Schwellung des Proc. mast, zu der sich nach wenigen Tagen Schüttelfrost mit intensivem nachfolgenden Fieber hinzugesellt. Incision der Geschwulst, Perforation des Knochens mittelst Troiscart's, Entleerung einer Quantitat Eiter, Pyimie, und Tod nach 10 Tagen.

7) Die 3 Falle von Kessel (auszinglich mitgetheilt im I. Heft des IV. Bandes dieses Archiv's p. 57). - Im 2ten Fall warde ein zu einer einjäbrigen Otorrhoe hinzugetretener Abscess des Proc. mast. durch Incision geöffnet und der Knochen durch die Sonde perforirt. Ebenso im 3ten Fall: Perforation durch Troiscart. In beiden: Heilung. - Lethal dagegen verilief der I. Fall. - Nach 11jährigem Bestehen einer Otorrhoe bei einem 25jährigen Bauern kamen die unzwcideutigsten Erscheinungen einer abscedirenden Entzündung des Proc. mast. hinzu. Trotzdem wurde aus nieht ersichtlichen Gruinden die Incision resp. Perforation 14 Tage lang verschoben, bis Pyämie hinzutrat. Section ergab : eitrige Sinus-Thrombose.

8) meine Beobachtung.

In sämmtlichen obigon Fälen, mit Ausnahme der Hinton'schen and dor meinigen waren neben deu ortlicher noch Cerebral-Symptome zugegen.

AdB. 1) Der Fall von Flaiz (dieses Archiv II, 228). Flaiz operirte mehrere Monate nach Beginn des acuten eitrigen TrommelhöhlenKatarrh's, obgleich zur Zeit der Pros. mast. weniger empfindlich und geschwollen war, als etwa 14 Tage früher, wo wiederholt durch Incision Eiterentlcerungen aus dea denselben bedeekenden Weichtheilen statţgefunden hatten, bei unerträglichen Schmerzen und nächtlichen Delirien; beiläufig ohne Riter sofort in den Zellon des Proc. mast. zu finden, aber mit nachträglicher Entleerung and ginstigem Erfolge.

2) Pagenstecher's I. Beobachtung. "Etwa vor 30 Jahren gelegent* lich der Masern begonnene eitrige Otitis media mit muthmasslich lange Zeit bestandener Caries unter der oberen Fläche des Felsenbeins. Tod durch eitrige Neningitis ohne eariöse Perforation oder eitrige Thrombose muthmasslich durch Fortleitung des Processes längs der Scheide des Acusticus; vollständige Sclerose des Proe. mast. und voraussichtlich erfolglose Perforation desselben 36 Stunden ror dem Tode. Mangel jeglicher auf entzindliche Vorgänge zu beziehender Symptome am Proc, mast. 
Unter allen diesen Beobachtungen hatte streng genommen allein die von Flaiz eme Aehnlichkeit mit den örtichen Verhältnissen bei meiner Kranken." Aber der von Flaiz behandelte Kranke batte cerebrale und febrile turscheinungen, die in meinem Falle fehlten. Ueberdies schien mir das Referat (l. c.), sofern der College nicht durch eigne Untersuchung den Thatbestand nach der Operation festzustellen Gelegenheit hatte, sondern lediglich durch die Berichte einer nichtärztlishen Persönlichkeit über das weitere Verbalten des Kranken etwas erfubr, doch zu mangelhaft, um obne Weiteres in gleicher Weise zu handeln.

Aus den angefuhrten Gründen bielt ich mich berechtigt, die Operation bis zu dem angegebenen Termine zu versehieben. Nach dem weitern Verlaufe der Sache glaube ich jetat für analoge Verhältnisse ein solches Benehmen, beziehentlich eine solche Verzögerung der Operation nicht mehr empfehlen zu dürfen. Die Antiphlogose hat sich bei meiner Kranken zur Hemmung des eitrigen Schmelzungsprocesses nutzlos erwiesen, und die der Kranken mittelbar aus der Verzögerung der Operation, beziehentlich dem langen Fortbestehen der Eiter-Retention und der entzündlichen Vorgänge innerhalb des Knochengewebes : erwachsenen Gefahren (Erysipel mit hochgradigem Fieber und intensiv entwiekelten Cerebral-Symptomen) wïrden durch zeitigere Operation höchst wahrscheinlich vermieden worden sein. Hiezu kommt, dass das operative Verfahren an sich bei einiger Vorsicht weder gefahrvoll nooi sehwierig ist. Verletzungen der Dura mater, des Sinus transversus werden durch den Läufer des akidopeirastischen Bohrers fast unmöglich gemacht. $\mathrm{Da}$ wahrscheinlich nicht wenigen Collegen das Instrument unbekannt ist, weil die Beschreibung desselben in einer nicht im Bucbhandel ersebienenen kleinen Abhandlung "Ueberblick über die Akidopeirastik, eino neue Untersuchungs-Methode mit Hilfe spitzer Werkzeuge von Prof. Dr. A. Th. Middeldorpf" enthalten ist, erlaube ich mir die betreffende Stelle jener herzusetzen und auf die Abbildung zu verweisen.

Fig. 1. "Explorativbohrer. Das Instrument ist dem Bohrex dcr Zahnärzte entlehnt und hat dic Vorzüge, dass man in der. Tiefe durch enge Oeffnungen hindurch in allen Richtingen operiren kann. Im motallenen Knopfo b. ist drehbar beweglich eingelassen der schraubeufồrmig $21 / 2$ mal gedrehte Sẹhaft (die Zeichnung gibt nur die $1 / 2$ Länge des Schraubentheiles an), welcher unten den mei"lnen Ansatz mit dem Einsehnitt $b$; trügt. 
In den Ansatz steckt man:

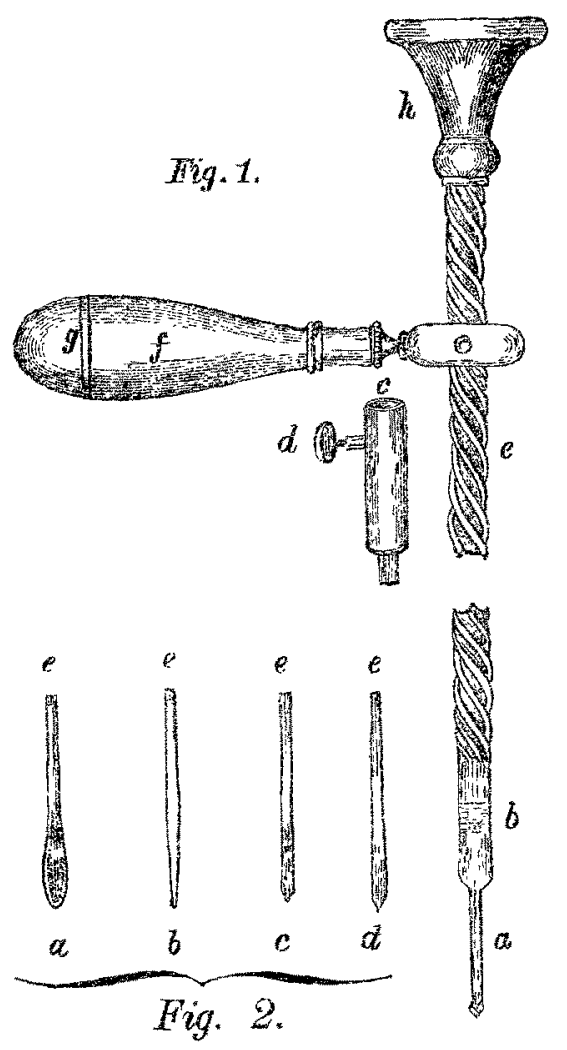

Fig. 2. Die Bohrspitzen a. b. c. d., die bei e. so quer abgefeilt sind, dass, um ihre Drehung zu hindern, die kleine Flache der Spitze sich auf die Fläche des Einschnitts b. legt.

Die Drehung der Bohrspitze nach rechts and links erfolgt durch Hinauf - und Hinab. schieben der mit einem Griffe gf. versehenen Mutterschraube g. Auf dem Ansatz b. und ther die Bohrspitze a. lasst sich rerschieben und durch die Schraubed. feststellen der Läu. fer cd, der das zu tiefe Winsinken des Bohrers hindert, gleichsam ein Abaptiston bildet. Fig. 2 as ist die Lôffolbolrrspitze, namentlich zw Schädel. Perforation geeignet, da sie die innere Glastafel ausschneidet, gieichsam nioht durehsticbt, also der Dura mater weniger gefăhrlich werden kann. Der Griff ist bei $g$. abschraubbar und enthält in seiner Hoblung die Bohrspitzen". -

Für die meisten Falle wird allerdings bei einiger Vorsicht auch ein einfacheres Bohrer- oder troiscartartiges Instrument, z. B. das von Schueurtze benützte oder selbst ein starkes Bistouri vollständig ausreichen; für diejenigen, wo eine bedeutende Selerose der zu durchbohrenden Wand rorausgeseizt werden muss, ein vollkommeneres unentbohrlich, für zweifelhaft erwinnscht sein und wie bereits dargethan, dïrfte das empfohlene die bisher benìtzten wobl ubertreffen. - Fur die seltenen Falle, die die Entfernung eines Sequester's erheischen, wird eine kleine Trepan-Krone stets ibre Bedeutung behalten.

Sohliesslich noch einiges über den zur Operation greeignetsten Termin. 
Dass man bei einer dem Abscediren der äussern Knochenwand naben, mit intensiven Schmerzen allein oder gleichzeitig mit Fieber, Cerebral-Erscheinungen etc.verbundenenEntzïndung des Warzenfortsatzes sofort durch eine Wilde'scheIncision, resp. Durchbobrung oder Durchschneidung der erweichten Knochendecke einzuschreiten hat, gilt unter Sachverständigen als selbstverständlich.

Wie aber soll man sich in Fallen verhalten, snalog dem von mir beobachteten? Soll man etwa sagè, die Erfahrungen seien noch nicht reif, darüber zu entscheiden? Das ist aus humanen Rücksichten unzulässig. - Denn es könnten leicht ein Paar Decennien vergehen, ehe diejenigen, welche so donken, die gewünschte Zahl von Beobachtungen gesammelt bätten. Mir scheint vielmehr, so weit ich entfernt bin, diese meine Ansicht zu einen unverbrüchlichen Axiom zu erheben, auch für zweifelhafte Falle der Art die Perforation indicirt, sobald durch eine fen individuelien Verhälinissen angepasste Antiphlogose nicht wenigstens ein allmähliges, durch Verringerung der objectiven Erseheinungen unzweidentig manifestirtes Rückgüngigwerden des Processes erwiesen ist.

Denn die mit dew operativen Verfahren verbundene Gefahr oder Sehwiorigkeit exseheint den deletären Folgen, einer eitrigen Thrombose, einer eitrigen Meningitis, den Zerstörungen des labyrinth's, den Corrosionen der grossen Gefässe u. s. w. gegentiber höchst geringtügig und der durch die rechtzeitige Operation herbeigefubrte ginstige Erfolg wach den bisherigen Erfabrungen fast zweifellos.

An die praktische Bedeutung meines Ansieht abor die eventuelle Anticipation des Operations-Termins wird man unwllkïlich beim Durchlesen der ersion Kessel'sehon Beobachtung genahnt. So weit es gestatiet ist, allein nach den Worten des vorliegenden Referats zu urtheilen, móchte man glauben, dass der beireffende Kranke durch eine in meinem Sinne rechtzeitige Operation gerettec worden wäre.

Wie es als ein unzweideutiger, durch Roser eingefübrter Fortschritt gilt, in operations-geeigneten Fallen von Croup nicht bis zur Asphyxie zu warten, sondern schon im Stadium der beginnenden Erstickungs-Noth zu operiren, so scheint es mir auch in den fraglichen Fälen mit unvollkommen entwickelten Lokal-Symptomen keineswegs zweckmässig, erst drängeode, z. B. cerebrale Erscheinungen abzuwarten. Aber auch in jenen, gewiss seltenen Fallen, wo die Zeichen eines entzündlichen oder cariösen Vorganges des Processus mast. vollständig' mangeln, wie in der ersten Pagenstecher'schen Beobachtung und lediglich die Anamnese neben dem Befunde eines chronischen eitrigen Trommelhöblen-Katarrh's odor einer beschrïnkten Caries des Meatus 
auditorius eine Caries des Felsenbeins vermuthen lassen, halte ich aus den bereits angefibhrten Gründen und weil so allein der Weg zur Heilung der Caries gebahnt wird, die Operation für angezeigt, noch ehe bertrohliche Erscheinungen eintreten. Bedïrfte es noch einer weitern Begründung, die in meinem Sinne rechtzeitige Operation zu empfehlen, so könnte ich mich beztiglich der verderblichen Folgen, welche durch langbestehende Eiterdepots in den. Höhlen des Mittelohrs erfahrungsgenüsss für den übrigen Organismus herbeigeführt werden, auf die' höchst lehrrejchen Beobachtungen von v. Tröltsch. (vd. Ohrenheilkunde pag. 366) und Schwartze's (dieses Archiv II. 4 280) beziehen. Das auffallend häufige Zusammentreffen der fraglichen Eiteransammlungen mit rapid verlaufender Tuberculose ist darch jene Autoren empirisch, durch Buhl und die verdienstrollen Arbeiten von Lebert und Wyss, Cohnheim, Wilson Fox, Waldenburg a. A. derartige Beziehungen im Allgemeinen experimentell als orwiesen zu betrachten.

Würde aber auch in einem Falle der fraglichen Art der cariöse Herd nicht gefundon werden, also das Verfahren ein akidopeirastisches Experiment gewesen sein, so wäre dasselbe mindestens ebenso berechtigt, wie etwa eine Tracheotomio oder Herniotomie bei nachträglich nicht glikcklich verlaufenden Fallen. - Hegen doch v. Tröltsch und Schwartze, wic ich aus des ersteren Lehrbuch (4. Aufl. S. 382) and aus einer brieflichen Mittheilung des letzteren entnehme, die. Ueberzeugung, dass die Perforation des Processus mastoideus in nicht fánger Zeit bei besondors bartnäckigen Eiterungen im Mittelohr beziehentlich zur Hoilung solcher gemacht werden wird, auch wenn noch keine drohenden Symptome von Eiter-Retention vorhanden sind. - In der That sind die oben citirten experimentellen Resultate von Buhl, Lebert, u. s. w. ebenso geeignet, ibre Voraussicht zu rechtfertigen, wie das Misslingen der sonstigen Cur zur Beseitigung des chronischen eitrigen Katarrh's trotz der Behandlung nach der ąnexkannt besten Methode die Voraussetzung gestattet, dass eine den Mitteln und Verfahrungsweisen unzugängliche Eiteransammlung innerbalb der Zellen des Warzenfortsatzes die Hartnäckigkeit des Zustandes bedingt.

\section{Hesultate aus den bisherigen Beobachtangen.} werden:

I) Die Operation ist indicirt und muss ohne Verzug gemacht

1) beim Vorhandensein von Erscheinungen, welche die Eiterung in Innern des Knochens mit Ueberausdehnung desselben beriebentlich 
entzündliche Erweichung der äussern Knochenschichte kennzeichnen bei gleichzeitigem oder fruher dagewesenem Eiterausfuss aus dem Gehörgange:

2) beì nur mässig entwickelten, auf entzündliche Frweichung des Knochengewebes des Processus mast. bezighlichen objectiven Symptomen, vorausgesetut, dass die begleitenden Erscheinungen, seien es intensive, örtliche und irradirte Behmerzen allein oder in Verbindung mit cerebralea $u_{0}$ s. w., der den individuellen Verhaltnissen anzupassenden doch nicht bis zum Fintritt drängender Symptome auszudebnenden Antiphlogose nicht gewichen sind;

3) auch da, wo, wie in der ersten Pagenstecher'schen Beobachtung; Zeicben der Theilnahme des Proc. mast. objectiv absolut mangein und nur ans dem begleiterden chron.-eitrigen Trommelhohlen-Katarrh oder beschränhter Caries des Meatus auditorius, der Anamnese u. s. W, die Vermuthung der Theilnahme des Felsenbeins gerechtertigt ist;

4) die Operation ist indieirt und darf gemacht werden, wenn ein chronischer eitriger Mittelohrkatarrh anch der anerkannt besten Bohandlungsmethode hartnäckig Trotz bietet, selbstredend voransgesetzt, dass die obne die fragliche Operation zu beseitigenden, der Heilung entgegenstehenden Hindernisse sämmtlich behoben sind.

II. Des Ort der Operation sei da, wo es sich voraussichtlich nur um eine Eiteransammlung innerbalb der Zellen des Warzenfortsatzes bandelt, der mithlere Theil, bei Caries des Felsenbeins mit Rucksicht auf die anatomische Continuitat die obere Grenze desselben,

III. Die Ausfuhrung der Operation geschieht in den sub 1 der Indicationen bexeichneten Fallen entweder mit einem starken Bistouri oder einem Troiscart oder bohrerartigen Instrument; in den sub 2,3 , 4 namhaft gemachten dagegen mit dem akidopeirastischen Bohrer *), der dureh die Leichtigkeit der Handhabung, die angemessene Klein heit der Bohrspitzen und vor Allom durch die Sicherheit gegen Nebenverletzungen den Vorzug verdient *s;).

Das genannte Instrument hat ausserdem das angenehme, dass es geeigneten Falls als Probebohrer benütat werden darf, zu welehem

*) Der akidopeirastisebe Bohrer wird znm Preise von 2/3 Thlr, von E. Püschel in Breslau (Weiden-Strasse 5) angefertigt.

* Zur Beschwichtigung ängstlicher Gemüther kann ich p̈brigens nicht umhin, nach einer briefichen Mittheilung meines verehrten Collegen Schwartze anzuführen, dass im Grund seiner Erfahrung selbst die Verjetzung dex Dura mater (Sinus transFersus) wiehts so bodenklich zu sein seheint, wie man fïrchten könnte. Fr fürchtete in einem solehen Falle den Tod und es trat nicht einmal Fieber ein. 
Zweeke allerdings unter Umständen auch starke Nadeln, Troiscarts zur probeweisen Flüssigkeits-Entnahme verwendet werden könnten.

Darüber, dass man, welchen Instrumentes man sich auch bedienen möge, in allen schwierigen Fallen dasselbe parallel der Richtung der äussern Gehbrgangs-Oeffnung wirken zü lassen babe, sind alle Autoren einig and die Nothwendigkeit, wo es sich um Durchbohrung bei Caries des Felsenbeins bandelt, aus Betrachtung des anatomischen Präpurates ersichflich.

IV. Die Prognose ist nach dem bis jetzt vorliegenden allerdings noch besehränkten statistisehen Material bei geeigneten Fällen günstigo zu stollen, vorausgesetzt, dass nicht unheilbare Consecutiv-Zustände, z. B. Pyâmie, bereits eingetreten sind, zumal technische Schwierigkeiten nicht vorhanden und üble Ereignisse mit Leichtigkeit zu vermeiden sind. Ist auch der primäre Eindruck der Operation nicht ein wo eclatanter wie der der Tracheotomie bei Croup, so treten die günstigen Folgen doch bimen Kurzem ein und babnen der definitiven Heilung den Weg. In nicht wenigen Fullen ist dieselbe als eine lebensrettende anzusehen und zweille ich nicht, dass wir uns der Zeit nähern, wo die Mehrzahl der Collegen in ähnlichem Masse wie der Tracheotomie bei Croup dem Verfabren ihre besondere Aufmerksamkeit zuwenden wird. - 